\title{
An enhancement of whale optimization algorithm using ANN for routing optimization in Ad-hoc network
}

\author{
Himani Yadav", Umesh Lithore and Nitin Agrawal \\ Department of Computer science and technology, NIIST, Bhopal, India
}

\section{(C)2017 ACCENTS}

\begin{abstract}
This work presents Ad-hoc optimal route finding with the help of a meta-heuristic algorithm from the group of swarm algorithms named as whale optimization algorithm (WOA) adding new functionality of artificial neural network. This is a multi-objective algorithm that can be helpful in solving many problems. This algorithm copies the hunting behaviour of the humpback whale which is one of the biggest animals on earth and it also includes the functionality of artificial neural network which is based on the concept of biological neural network. Whale optimization algorithm inherits the spiral net technique from hump back whales, in which the whale creates a bubbly spiracle net for encircling the pray and then find the prey either by applying the best find strategy or randomly. This algorithm can be used in many fields like ad-hoc, power electronics, wireless network and classical engineering problems. But here in this paper it is going to apply on route finding problem in ad -hoc network. Where the ad hoc network require to find the location of moving device which keep changing its position and also needed to find the best path from one device to another for data delivery. In such type of problems whale optimization algorithm can be used to produce the batter results then tradition algorithm.
\end{abstract}

\section{Keywords}

WOA algorithm, MANET, Artificial neural network, Route finding.

\section{Introduction}

A term MANET/WSN is utilized for Mobile specially appointed system which is autonomous structure of compact hubs associated by remote connections in an independent form in a bigger system. Hubs can interface, separate and re-associate as the time goes on and all the time the system associations will work between the hubs that are a piece of it. Directing is a procedure of choosing the best path inside the network. Beforehand, the term routing also supposed forwarding network traffic amongst network. All things considered, that last perform is more beneficial portrayed as sending [1]. Routing is performed for a great deal of sorts o1f system, together with the phone arrange (circuit exchanging), computerized information systems (such in light of the fact that the web), and transportation network. The different problems which can rise in packet delivering such as ordering of packet receiving and ordering of packet transfer in MANET. Keeping up the requesting of parcel is difficult to keep in genuine system multi cast routing.

*Author for correspondence
Another issue, for example, loss of packet while conveying different quantities of substantial packets in a single endeavour or in overwhelming blockage arrange, such things furthermore causes organize packet delay[2].

A cellular network is made effectively by free of cell centre points which can be associated through Wi-Fi hyperlinks with no using the present set up or government organization. The center points can wholeheartedly move randomly and create themselves conflictingly; thusly, the frameworks remote topology can change dependably and sporadically. These frameworks can work in a freeway, or can be related with the far reaching Internet. Adaptable uncommonly selected framework is establishment less framework so they don't require any settled system. Each center point will have the ability to talk about particularly with any center to live enveloped by its broadcast range [3].

The scope region of cell systems is isolated into cells, every phone having its own particular reception apparatus for transmitting the signs. Every cell has its own frequencies. Information correspondence in cell systems is served by its base station transmitter, collector and its control unit [4]. 


\subsection{MANET/WSN challenges}

The capacities of MANET present a few requesting challenges that should be examined warily sooner than a broad business sending can be normal. Those include:

1) Routing

2) Security and Reliability

3) Quality of Service (QOS)

4) Internet working

5) Energy consumption

\subsection{Related work}

Whale optimization Algorithm is based on whales, which use their social behaviour for finding optimal solution for problem. WOA is a swarm based algorithm which is getting very popular in now days [5].

Whales are very special creature. They are known as the biggest living animal on earth. Not only biggest, they are very intelligent to. They have some similar cell in their mind like humans. That is known as "spindle cell". Spindle cell is responsible for social behaviour, learning, grouping, making community, supporting. In a single word we can say that spindle cell is responsible for intelligence. Which make us different from others and this spindle cell is twice in whales then an adult man. Because of that whales can learn, make groups, can make judgment, lives in community and work together. With all these hump back whale have a special and the different way of feeding. There way of hunting is different from all others whales and fishes [6].

Whales don't sleep. As they have to breath from the surface of water. With the same reason the humpback whale hunt the fishes that swim near the surface of water. On the basis of these characteristics of the whales a meta-heuristic algorithm can be form to provide various optimal solutions for the optimization problems. That provides an improved and batter performance as compare to various techniques. We have included artificial neural network with basic WOA to get the batter result [7].

\subsection{A fast, three-layer neural network for path finding}

A way arranging calculation is proposed to discover a way in light of neighborhood rules connected to a three-layer artificial neural system. Each layer comprises of two-dimensionally masterminded neurons with intermittent associations (recurrent connections) inside a restricted neighborhood. The yield of one layer decides the weights of the associations in the following layer. On a basic level, the technique depends on a dissemination procedure, however is changed with the end goal that it doesn't experience the ill effects of a few downsides engaged with this calculation. By use of a nonlinear change in layer 2, the dissemination front has the subjective properties of a propagation wave. In this manner, constrained determination of the units isn't basic, rather than established dispersion calculations. Besides, the calculation by and large does not experience the ill effects of the superposition of dispersion angles when a few ways are conceivable. The dispersion happens in a space secured with 'obstacle potentials' which diminish the speed of the diffusion front. Along these lines the way can keep up a movable wellbeing edge in connection to the snags, for instance, to adapt to issues of fragmented information of the obstacle potentials. The calculation along these lines joins the upsides of the dispersion calculation, specifically evasion of neighborhood minima, of wave spread, i.e. adapting to restricted determination, and the potential field approach, i.e. keeping up a security edge in connection to obstructions. The dispersed design additionally takes into account 'spatial insertion' between the units (coarse coding), accordingly giving smooth way shapes. A correlation with ways created by human subjects demonstrates some closeness on the subjective level; however there are additionally clear contrasts [8].

\section{Proposed methodology}

In existing procedures there are different variations of WOA are displayed. Yet, in this system different routes are considered to give arrangement. Consequently choice component is required to choose advanced routes to transmit packet. That process increases the waiting time to route packets that increases the delay because it consumes too much time which degrades the performance of the whole technique. In there are numerous connections introduced to associate one hub to other, when an optimal routes chose some other most limited routes additionally chose with that which likewise expands the standardized directing proportion [9].

A new technique is required to solve all the routing related issues in existing techniques. And here is the proposed algorithm Based on ANN and WOA.

\subsection{Proposed algorithm}

A pseudo code is presented here, which is based on proposed model and Figure 4 is the graphical representation of algorithm: 
1. Setup the complete environment and configurations.

2. Initialize the Whales population $x i=(i=1,2 \ldots n)$ and further initialize all the nodes .

3. $\mathrm{X}^{*}$ the best search agent.

4. Initialize neural network function as the term of energy, trust and hop count.

5. While $(\mathrm{t}<$ Maximum no. of iterations for each search agent)

6. Update the position, random vector, distance, coefficient vector (a,A,C,L,P)

7. For each

Produce new solutions through adjusting NFC, by modifying values, and updating energy, trust and hop.

8. If1 $(\mathrm{p}<0.5)$

9. If $2(/ \mathrm{A} /<1)$

10. Update the position of current agent $\mathrm{X}(\mathrm{t}+1)=\mathrm{X}(\mathrm{t})-\mathrm{A} *\left\{/ \mathrm{C}^{*} \mathrm{X}(\mathrm{t})-\mathrm{X}(\mathrm{t}) /\right\}$

11. Else If $2(/ \mathrm{A} />=1)$

12. Select random search agent (Xrand) update the position of current agent $\mathrm{X}(\mathrm{t}+1)=\mathrm{Xrand}-\mathrm{A} *\left\{/ \mathrm{C}^{*} \mathrm{Xrand}-\mathrm{X} /\right\}$

13. End If 2

14. Else If1 $(\mathrm{P}>=0.5)$

15. Update the position of current agent $\mathrm{X}(\mathrm{t}+1)=\mathrm{D} * \exp (\mathrm{b} * 1) * \cos (2 * 3.14 * 1)+\mathrm{X}(\mathrm{t})$

16. End If1

17. Execute the value of neural function (NFC) and calculate the fitness of each search agent based on Max NFC value

18. End For

19. Update $X^{*}$ if there is better solution

20. $\mathrm{t}=\mathrm{t}+1$

21. End While

22. return $X^{*}$

The below flow diagram shows the complete details on how the experiment is performed and executing in a manner.

\section{Experiment and result}

To implement proposed technique, NS2 simulator is used which provides an enhanced functionality to develop research projects for communication network. In that way it provides a framework to develop such projects [10].

Network simulator is used to analyse the standard WOA and modified WOA. The existing WOA is run on this simulator and with same environment this simulator will again run for Modified WOA to show the comparison of performance on parameters: packet delivery ratio (PDR), throughput, end-to-end delay, normalized route load and energy. The modified technique is simulated with following scenarios shown in Table 1:

Table 1 Simulation Scenarios

\begin{tabular}{ll}
\hline No. of Nodes & 30 \\
No. of Source & $1 \mathrm{~s}$ \\
Area & $1000 \mathrm{X} 1000$ \\
Mobility model & Random waypoint \\
Bandwidth & $2 \mathrm{mbps}$ \\
Speed & $0,1,5,10,15,20 \mathrm{~m} / \mathrm{s}$ \\
Pause time & $10 \mathrm{sec}$ \\
Buffer Size & 100 \\
Transmission & $2100 \mathrm{~m}$ \\
range & \\
Sensing range & $2100 \mathrm{~m}$ \\
Packet size & 1012 bytes \\
Traffic source & Constant Bit Rate (CBR) \\
MAC protocols & IEEE 802.11 \\
\hline
\end{tabular}

\subsection{Result analysis}

This section shows a comparison analysis for the results for proposed and existing technique.

Evaluation Parameter: the evaluation parameters are some perimeters that are used to calculate the result. They are normalized routing load; packet delivery ratio (PDR), throughput, end-to-end delay and energy are used to calculate performance of technique.

\subsection{Normalized routing load}

Routing load over the various nodes is calculated to find and manage the load over the in MANET.

\subsection{Throughput}

Throughput is the measure of work in a given time frame. That is the final output produced by the whole process. It is the measure of complete performance of the technique which uses to measures the performance of the techniques.

\subsection{PDR (Packet Delivery Ratio)}

It is the ratio of, no. of packet accurately delivered to the destination in a time unit.

\subsection{Average delay}

Average delay is the time taken by receiver in sending the response of packet received from sender.

A graphical analysis is shown in graph for the proposed technique, which shows a graphical comparison between the tradition wheal optimization and proposed algorithm. Here Table 2 shows the comparison of ppacket Loss Ratio between WOA and Modified WOA and Figure 1 shows the graphical 
comparison of packet Loss Ratio between WOA and Modified WOA.

Table 2 Packet loss ratio (PDR)

\begin{tabular}{lll}
\hline $\begin{array}{l}\text { Simulation } \\
\text { Time }\end{array}$ & WOA & Modified WOA_ANN \\
\hline 10 & 0 & 42 \\
20 & 84 & 12 \\
30 & 60 & 98 \\
40 & 22 & 70 \\
50 & 72 & 18 \\
60 & 62 & 26 \\
70 & 68 & 38 \\
\hline
\end{tabular}

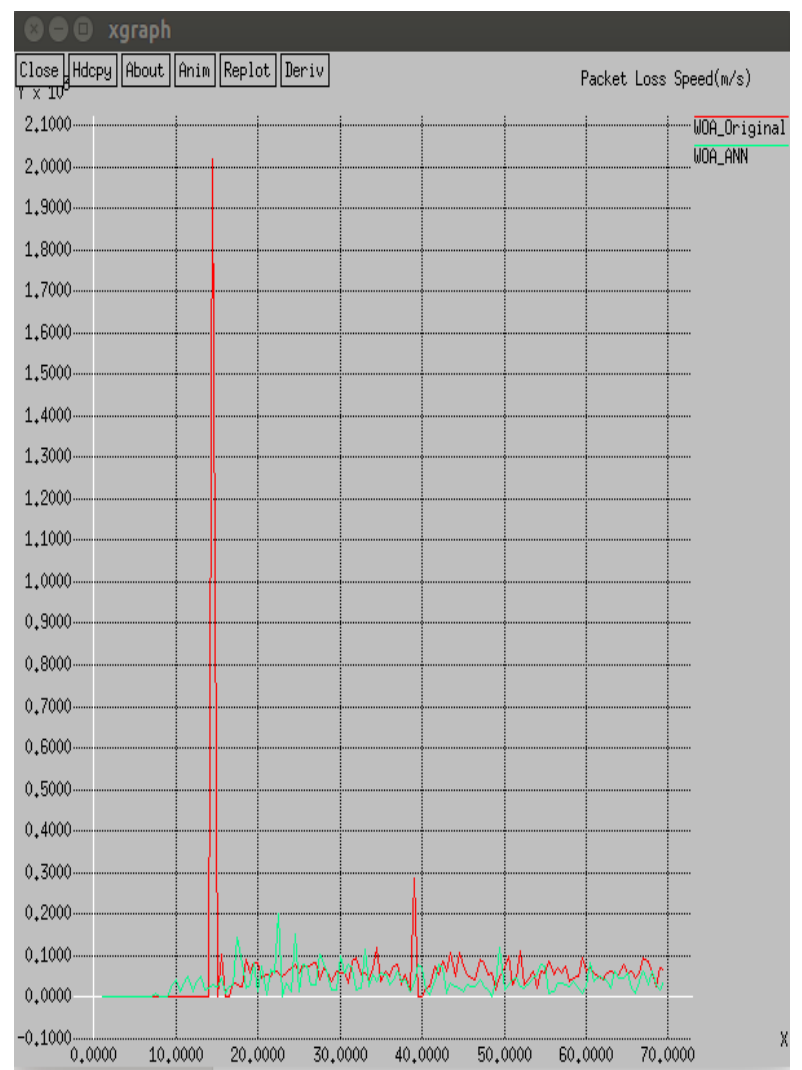

Figure 1 Packet loss speed (PLS)

3.6End-to-end delay vs. speed

It is the time taken for a packet to be transmitted in the network from source to goal. Table 3 shows the comparison of end to end delay between WOA and Modified WOA. And Figure 2 shows the graphical comparison of end to end Delay between WOA and Modified WOA

End-to-end delay $=\sum$ (arrive time-send time $) / \sum$ Number of connections.

Table 3 End to end delay

\begin{tabular}{lll}
\hline $\begin{array}{l}\text { Simulation } \\
\text { time }\end{array}$ & WOA & $\begin{array}{l}\text { Modified } \\
\text { WOA_ANN }\end{array}$ \\
\hline 10 & 0 & 1.2 \\
20 & 2.26 & 2.9 \\
30 & 3.03 & 2.6 \\
40 & 2.85 & 1.22 \\
50 & 2.01 & 1.79 \\
60 & 2.34 & 1.77 \\
\hline
\end{tabular}

3.7Throughput vs. speed

Network throughput is the average number of successful message sent over a communication network. This data can be transmitted over the physical or logical link. Transmission Time $=$ packet Size / Bandwidth (sec) Throughput = packet Size / Transmission Time (kbps) If the packet delivery ratio increases, the impact of it clearly shows on throughput that increases too.

Here Table 4 shows the comparison of Throughput between WOA and Modified WOA and Figure 3 shows the graphical comparison of throughput between WOA and Modified WOA.

Table 4 Throughput

\begin{tabular}{lll}
\hline $\begin{array}{l}\text { Simulation } \\
\text { Time }\end{array}$ & WOA & $\begin{array}{l}\text { Modified } \\
\text { WOA_ANN }\end{array}$ \\
\hline 10 & 0 & 5875 \\
20 & 3427 & 3264 \\
30 & 3019 & 3182 \\
40 & 3182 & 5548 \\
50 & 3508 & 5222 \\
60 & 3672 & 3998 \\
\hline
\end{tabular}

A depiction over the execution situation to actualize proposed procedure and assessment of the consequences of the strategy is exhibited. On the assessment exhibited in result investigation segment, assessment over all the parameter demonstrates that, proposed method gives better outcomes as contrast with the current strategy. 
International Journal of Advanced Technology and Engineering Exploration, Vol 4(36)

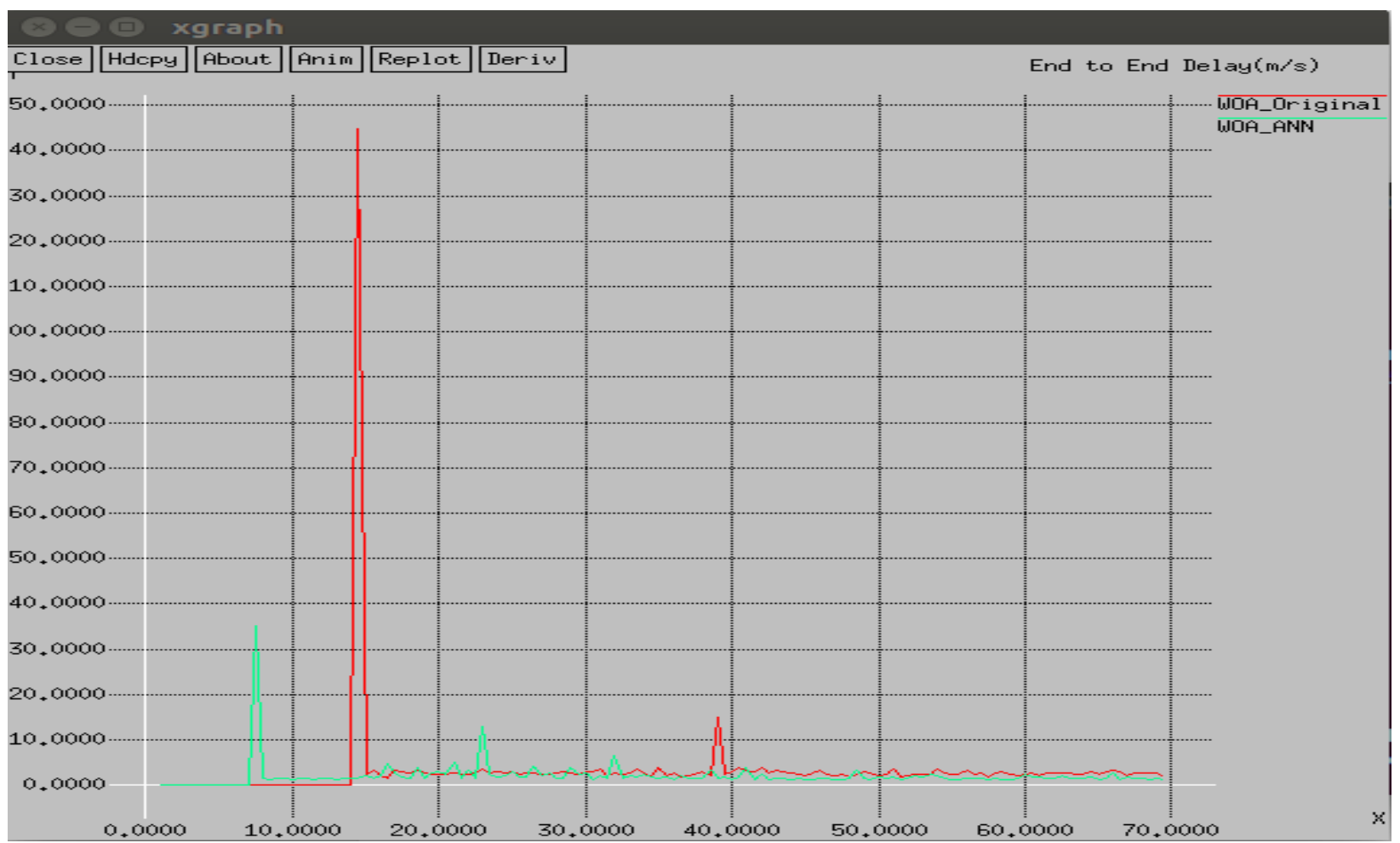

Figure 2 End-to-end delay vs. speed

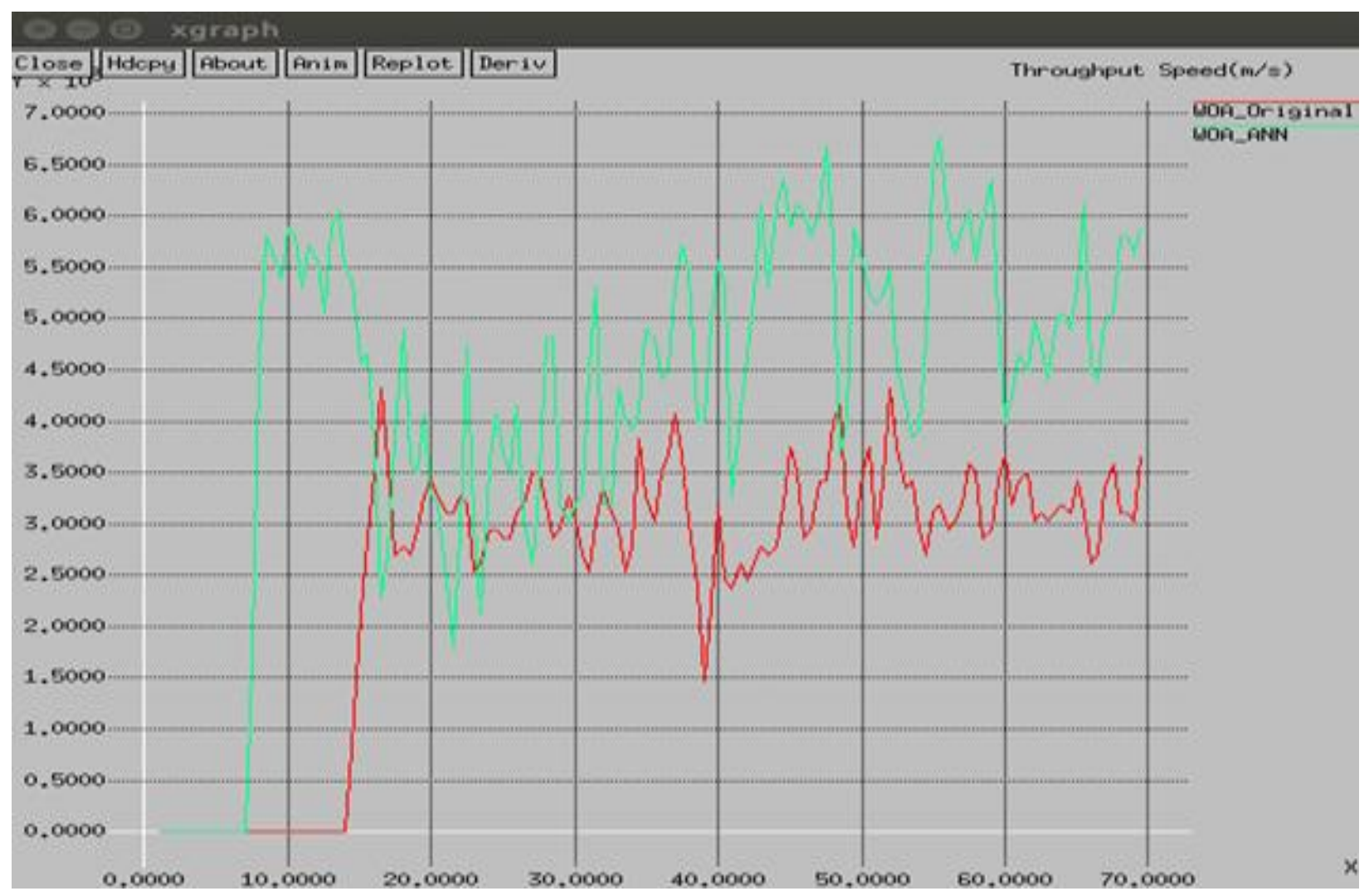

Figure 3 Throughput speed 
Himani Yadav et al.

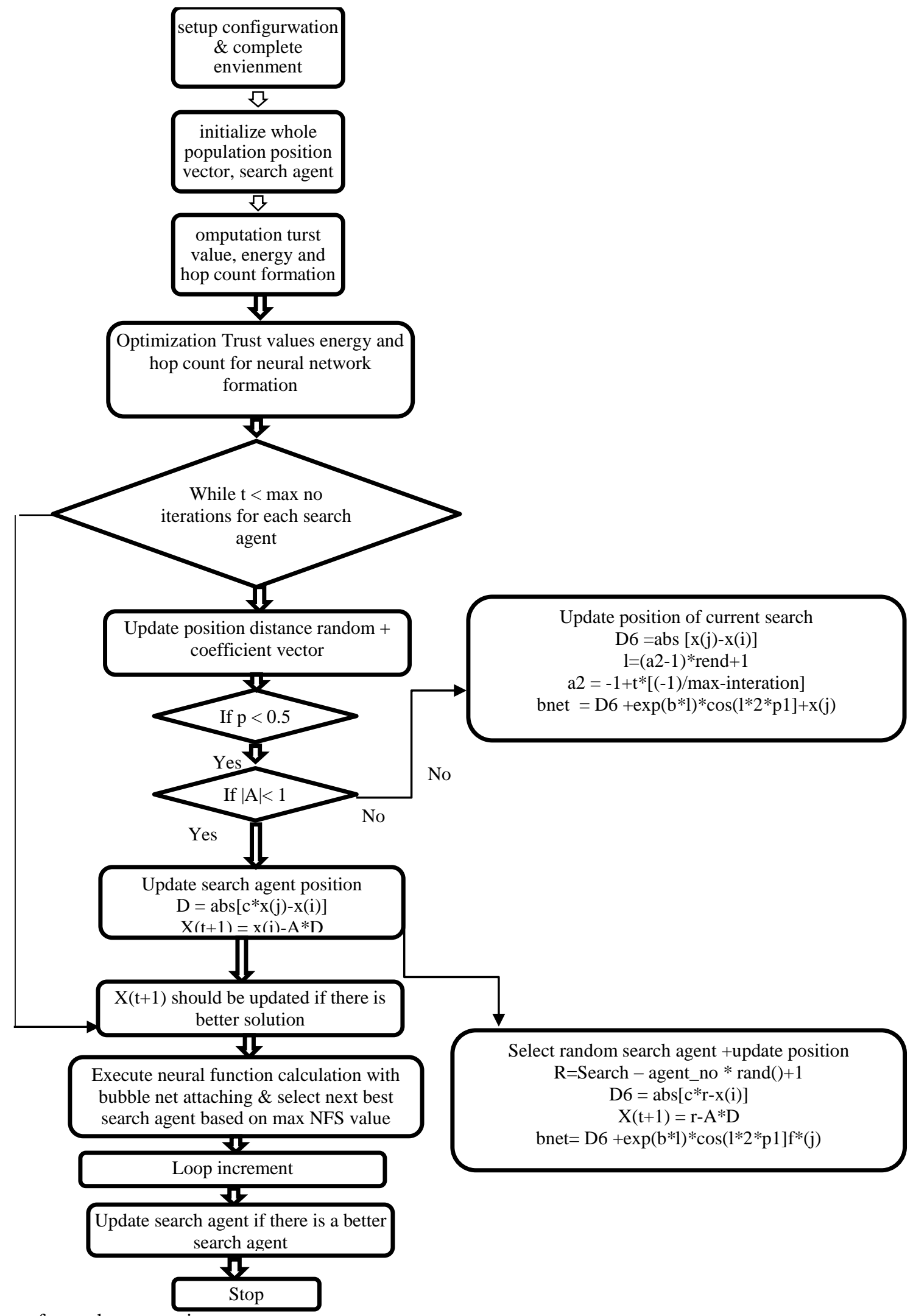

Figure 4 Flow diagram of complete scenario 


\section{Conclusion}

A network in which several mobile devices are connected to communicate over a wireless channel is called mobile ad-hoc network (MANET). In MANET the devices are in movable form, that's why the network topology changes frequently. In such type of network the routing is one of the hardest tasks to do, issues like congestion, packet loss and some others are very often to occur. WOA is a processing technique which delivers packet from source to destination, in this technique each node contains the table of links which contains the information about the different links to the different nodes. But in that method we require a selection mechanism for selecting optimum route from the multiple route provided in the table. For which we have provided WOA with the concept of artificial neural network. It updates the input perimeters with weight, which is an obvious phenomenon for ad-hoc network that keep changing the positions.

Our proposed work is based on the weight which gets updated with the help of ANN function. And then it applied on WOA. Thais change makes the algorithm more applicable for mobile ad hoc network.

\section{Acknowledgment}

None.

\section{Conflicts of interest}

The authors have no conflicts of interest to declare.

\section{References}

[1] Tan KC, Chew YH, Lee LH. A hybrid multi-objective evolutionary algorithm for solving truck and trailer vehicle routing problems. European Journal of Operational Research. 2006; 172(3):855-85.

[2] Ahirwar GK, Goyal S, Mishra N, Agrawal R. An enhancement of BAT algorithm using diversity function for routing optimization in WSN. International Journal of Control Theory and Applications. 2017; 10 (13): 255-64.

[3] Alba E, Dorronsoro B. Cellular genetic algorithms. Springer Science \& Business Media; 2009 Apr 5.

[4] Martin R, Stephen W. Termite: A swarm intelligent routing algorithm for mobile wireless Ad-Hoc networks. Stigmergic Optimization. 2006:155-84.
[5] Zeng B, Gao L, Li X. Whale swarm algorithm for function optimization. arXiv preprint arXiv:1702.03389. 2017.

[6] Mirjalili S, Lewis A. The whale optimization algorithm. Advances in Engineering Software. 2016; 95:51-67.

[7] Yadav H, Lilhore U, Agrawal N. A survey: whale optimization algorithm for route optimization problems. Wireless Communication. 2017; 9(5):105-8.

[8] Kindermann T, Cruse H, Dautenhahn K. A fast, threelayer neural network for path finding. Network: Computation in Neural Systems. 1996; 7(2):423-36.

[9] Archetti C, Speranza MG, Savelsbergh MW. An optimization-based heuristic for the split delivery vehicle routing problem. Transportation Science. 2008; 42(1):22-31.

[10] Hongping $\mathrm{Hu}$, Yanping Bai, and Ting Xu. Improved whale optimization algorithms based on inertia weights and theirs applications. 2017; 11:12-26.

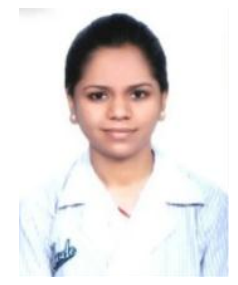

Himani Yadav has completed Computer Science Engineering from UIT RGPV in 2014. Currenly she is pursuing M.Tech from NIIST, Bhopal, India in the field of wireless communication. Area of interest is in the field of Database Management, Wireless Communication and Artificial Intelligence. Email: himaniyadav786@yahoo.com

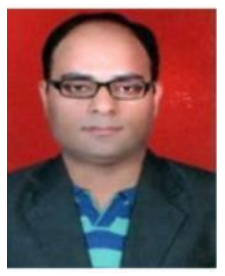

Dr. Umesh Lilhore is working as a Dean Research in the department of Computer Science at NIIST, Bhopal. $\mathrm{He}$ is $\mathrm{PhD}$ in Computer Science and M.Tech Computer Science and Engineering from RGPV, Bhopal, India. His current research interests include Cloud Computing, Machine Learning and Network Security.

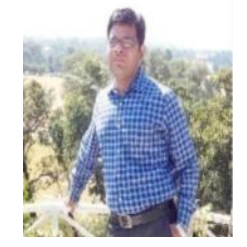

Nitin Agrawal is Assistant Professor in the department of Computer Science at NIIST, Bhopal. He is M.Tech from NIIST, Bhopal and MCA from UIT RGPV, Bhopal, India. His current research interests include Artificial Intelligence, Networking and Wireless Communication. 